\title{
Factors Predicting the Effects of Hybrid Assistive Limb Robot Suit during the Acute Phase of Central Nervous System Injury
}

\author{
Hideo CHIHARA, ${ }^{1}$ Yasushi TAKAGI, ${ }^{1}$ Kazunari NisHINO, ${ }^{2}$ Kazumichi YosHIDA, ${ }^{1}$ \\ Yoshiki ARAKAwA, ${ }^{1}$ Takayuki KIKUCHI, ${ }^{1}$ Yohei TAKENOBU, ${ }^{1}$ \\ and Susumu MiYAMOTO ${ }^{1}$
}

${ }^{1}$ Department of Neurosurgery, Kyoto University Graduate School of Medicine, Kyoto, Kyoto; ${ }^{2}$ Department of Rehabilitation, Kyoto University Hospital, Sakyo-ku, Kyoto

\begin{abstract}
To improve the activities of daily living of patients with injury to the central nervous system, physical therapy starting from the acute phase of the injury is important. Recently, the efficacy of physical therapy using a hybrid assistive limb (HAL) robot suit was reported. However, individual differences exist in the effects of HAL. We investigated factors predicting the effects of HAL in 15 patients at our institution with central nervous system injury, primarily due to stroke, who underwent training using HAL during the acute phase. Patients were classified as either "with HAL suitability" or "without HAL suitability" based on scores from 10-m walking speed, gait, satisfaction, and pain. In both groups, Brunnstrom stage before HAL intervention, Fugl-Meyer assessment (FMA), stroke impairment assessment set (SIAS), and functional independence measure (FIM) were evaluated. Although motor function items did not differ significantly, FIM cognitive function items $(P=0.036)$, visuospatial perception items on SIAS $(P=0.0277)$, and pain items on SIAS $(P=\mathbf{0 . 0 1 2 2})$ differed significantly between groups. These results indicated that training using HAL does not involve pain in patients with central nervous system injury during the acute phase, and exhibits positive effects in patients without pain and with high communication ability and visuospatial perception function. When conducting HAL intervention, incorporating functional assessment scores (FIM and SIAS), including peripheral items, may be useful to predict the suitability of HAL.
\end{abstract}

Key words: robot suit, HAL, rehabilitation

\section{Introduction}

Currently, hemiparesis caused by various brain and spinal cord injuries, including stroke, is the primary factor leading to the need for long-term care. Proactive physical therapy is thus recommended with the purpose of promoting patient independence in activities of daily living and reducing the amount of care. Moreover, in addition to traditional physical therapy methods, various intervention methods such as transcranial magnetic stimulation, ${ }^{1}$ electrical stimulation, ${ }^{2}$ and mirror therapy ${ }^{3}$ to reconstruct the neural network and plasticity of injured cranial nerve function have been assessed and have demonstrated positive effects. ${ }^{4}$ Swayne et al. described a theory of the stages of motor paralysis recovery as one of the founding concepts of the above methods, and stated that recovery is dependent on the excitability of the

Received August 18, 2015; Accepted October 21, 2015 remaining corticospinal tract and of the intracortical network during the acute stage after onset.

At our institution, we utilize the hybrid assistive limb (HAL) robot suit. Efficacy of physical therapy using HAL has been reported in patients with chronic phase spinal cord injury ${ }^{5-7}$ or after spinal cord surgery, ${ }^{8}$ as well as in stroke patients in the recovery ${ }^{9}$ or chronic phase. ${ }^{10}$ Additionally, some reports have described the feasibility and safety of this method in acute-phase stroke. ${ }^{11,12}$ However, in patients who are undergoing physical therapy using HAL, some show good results and some do not. The present study investigated factors influencing the good results of HAL on patients.

\section{Materials and Methods}

Robot suit HAL was introduced to our institution in December 2014. Since then, we have been conducting physical therapy using HAL primarily 
in patients with acute-phase stroke. The present study excluded patients who were able to use HAL only once due to early transfer to another hospital or patients who dropped out of the study due to exacerbation in general condition; and investigated a total of 15 patients ( 8 men, 7 women). This study group included patients who were 12 to 83 years old (mean age, 48.3 years) with the following primary diseases: hypertensive intracerebral hemorrhage $(\mathrm{n}=6)$, intracerebral hemorrhage due to arteriovenous malformation rupture $(\mathrm{n}=1)$, subarachnoid hemorrhage $(n=1)$, intracerebral hemorrhage associated with brain tumor $(\mathrm{n}=1)$, cavernous hemangioma $(\mathrm{n}=1)$, brain tumor $(\mathrm{n}=2)$, or spinal cord tumor $(\mathrm{n}=3)$ (Table 1). Intervention with therapists and training with HAL began 0-35 days (mean, 5.4 days) and 2-57 days (mean, 18 days), respectively, after onset or after tumor-extraction surgery. The study group performed HAL training 2-14 times (median, 6 times) during hospitalization at our institution.

\section{HAL training}

HAL was performed in accordance with the guidelines of the Ethics Committee of Kyoto University Graduate School of Medicine. Prior to HAL intervention, consent was obtained from patients after providing explanations using pamphlets and videos. Intervention with the single-joint-type HAL in a lying down or sitting position was employed first in patients who were unable to stand. In addition, when conducting standing and walking exercises, blood pressure was measured before and after HAL usage to confirm whether orthostatic hypotension was present. Training with HAL was limited to 2-3 times a week in consideration of patient endurance, and was used in combination with conventional physical therapy. HAL for living support (lower limb type, non-medical) was primarily used in the study group. In the present study, patients put on HAL in a sitting position, and performed standing training and gait training on a flat surface or treadmill (Fig. 1). During gait training, a monitor was displayed in front of the patient to provide visual feedback on the center of gravity and posture balance (Fig. 2).

\section{Assessments}

To extract patients who exhibit marked effects due to HAL usage, the effects of HAL were scored in the present study ("HAL suitability score"). The following four items, summed to a maximum score of 10, were evaluated: increase in $10-\mathrm{m}$ walking speed, gait improvement, patient and family member satisfaction, and pain development between pre-HAL intervention and completion of HAL training.

Ten-meter walking speed was assessed at the completion of HAL training and scored as follows: 0 , not able to walk $10 \mathrm{~m}$; 1 , improvement of less than 1.5-fold; and 2, showing 1.5-fold or greater

Table 1 List of cases

\begin{tabular}{|c|c|c|c|c|c|}
\hline Case & $\begin{array}{c}\text { Age } \\
\text { (years) }\end{array}$ & Sex & Diagnosis & Location & Suitability \\
\hline 1 & 59 & $\mathrm{~F}$ & Putaminal hemorrhage & Right & + \\
\hline 2 & 33 & M & Putaminal hemorrhage & Left & + \\
\hline 3 & 17 & M & Cortical hemorrhage (arteriovenous malformation) & Left & + \\
\hline 4 & 55 & M & Subarachnoid hemorrhage(anterior communicating artery aneurysm) & - & + \\
\hline 5 & 23 & $\mathrm{~F}$ & Cavernous malformation & Right & + \\
\hline 6 & 68 & M & Brain tumor (anaplastic astrocytoma) & Right & + \\
\hline 7 & 61 & $\mathrm{~F}$ & Brain tumor (metastatic tumor) & Left & + \\
\hline 8 & 39 & M & Spinal tumor (meningioma) & Spine (Th1-4) & + \\
\hline 9 & 70 & $\mathrm{~F}$ & Spinal tumor (meningioma) & (Th7-8) & + \\
\hline 10 & 12 & M & Spinal tumor (meningioma/ependyoma) & $(3-6)$ & + \\
\hline 11 & 81 & $\mathrm{~F}$ & Thalamic hemorrhage & Right & - \\
\hline 12 & 64 & M & Thalamic hemorrhage & Left & - \\
\hline 13 & 45 & $\mathrm{~F}$ & Putaminal hemorrhage & Right & - \\
\hline 14 & 15 & M & Putaminal hemorrhage & Right & - \\
\hline 15 & 83 & $\mathrm{~F}$ & Putaminal hemorrhage & Left & - \\
\hline
\end{tabular}

+: with suitability, -: without suitability, F: female, M: male. 

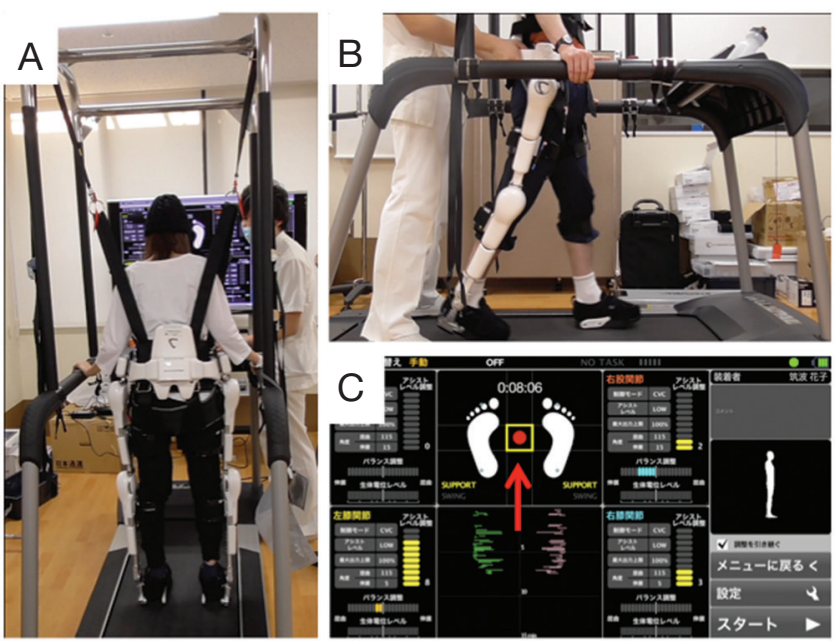

Fig. 1 Training using hybrid assistive limb (HAL) for living support (non-medical) with a treadmill. View from behind (A) and side (B). Training is performed under constant supervision by a professional physiotherapist, who adjusts the HAL-assistance setting and provides guidance on gait movements while observing the monitor and gait. The HAL monitor (C) is displayed in front of the treadmill and used to provide visual feedback to patients. Red arrow $(\uparrow)$ indicates the patient's center of gravity as obtained from the foot sensor.

improvement. Gait evaluation was conducted by two expert physical therapists using image analysis software and was scored from 0-3, ranging from exacerbation, unchanged, to marked improvement. Satisfaction was scored from $0-3$ by conducting interviews with patients and family members upon completion of HAL training. Pain was scored according to the usage of analgesic agents: 0, pain that requires analgesics; 1 , mild pain not requiring analgesics; and 2, no pain.

We retrospectively investigated the four items from the medical records. In our study group, this HAL suitability score ranged from 1-10 (mean, 6.93; median, 7). We divided patients into those with a suitability score of 7 or greater ("with HAL suitability") and those with a score of 6 or lower ("without HAL suitability"), and assessed related factors.

Items for evaluation were Brunnstrom stage (Brs) before HAL intervention, Fugl-Meyer assessment (FMA), and stroke impairment assessment set (SIAS) as indices to measure function; and functional independence measure (FIM) as an index to measure patient independence in everyday living.

\section{Results}

No significant differences in age, sex, or physique were seen between "with suitability" and "without
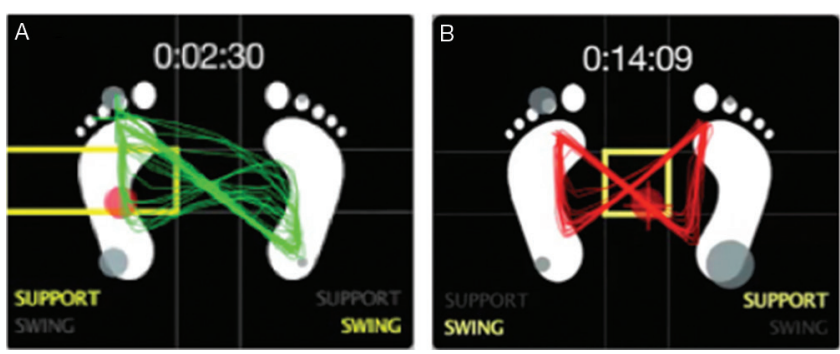

Fig. 2 Example of changes in assistance adjustment to the trajectory of the center of gravity in the "symmetrical butterfly shape." A: Trajectory without assistance, B: trajectory with assistance.

suitability" groups in this study. The number of days of until starting HAL training tended to be slightly greater in the "with suitability" group, but a significant difference was not evident. Number of HAL training sessions was also not significantly different. Regarding motor function before HAL training, lower limb Brs, FMA, and SIAS (lower limb-related items) all tended to be greater in the "with suitability" group, but again without statistical significance. Moreover, the motor function item on FIM also tended to be greater in the "with suitability" group, with no statistical significance.

In contrast, peripheral items associated with HAL training such as visuospatial perception and pain on SIAS and cognitive function items on FIM were significantly greater in the "with suitability" group (Table 2).

\section{Discussion}

Robot suit HAL is a powered exoskeleton developed by the Center for Cybernics Research at the University of Tsukuba. In HAL, biopotential signals that reach the muscle via efferent neurons of the spinal and peripheral nerves from the brain are detected at the skin surface when the individual decides to move the four limbs, and the exoskeleton is controlled based on these signals. Muscle movement is supported by the exoskeleton, and is transmitted to the brain via afferent neurons. The arrangement of this interactive feedback through HAL is postulated to efficiently excite the remaining corticospinal tract in the injured brain and spinal nerves and enhance the effects of physical therapy. Additionally, the cycle of voluntary movement from afferent signals in the brain is postulated to heighten the excitability of the existing intracortical network and promote the formation of new networks. Moreover, in HAL, not only the effects of feedback from the biopotential signals were detected at the skin surface, but also the 
Table 2 Comparison of "with hybrid assistive limb (HAL) suitability" and "without HAL suitability

\begin{tabular}{|c|c|c|c|}
\hline Groups & $\begin{array}{l}\text { With HAL suitability } \\
\qquad(\mathrm{n}=10)\end{array}$ & $\begin{array}{l}\text { Without HAL suitability } \\
\qquad(\mathrm{n}=5)\end{array}$ & $\mathrm{P}$ \\
\hline Age & $12-70(43.7)$ & $15-83(57.6)$ & 0.2639 \\
\hline Sex (M:F) & $4: 6$ & $3: 2$ & 0.4642 \\
\hline Height (cm) & $150-178.4(162.7)$ & 139.3-165.8 (156.4) & 0.2449 \\
\hline Weight (kg) & $29-76(52.4)$ & $44-74.2(56.76)$ & 0.5405 \\
\hline Interval until HAL intervention (days) & $2-57(20.1)$ & $5-21(13.6)$ & 0.3392 \\
\hline Number of HAL training sessions & $3-14[7]$ & $2-11[6]$ & 0.5837 \\
\hline Brs before HAL training: lower limb & $1-6[4]$ & $2-4[3]$ & 0.5731 \\
\hline FMA & $66-221[210]$ & $94-184[133]$ & 0.233 \\
\hline Total SIAS & $16-68[61]$ & $14-49[38]$ & 0.2414 \\
\hline SIAS: lower limb & $7-29[22]$ & $9-24[16]$ & 0.3875 \\
\hline SIAS: pain & $3[3]$ & $0-3[2]$ & $0.0122^{*}$ \\
\hline SIAS: visuospatial perception & $2-3[3]$ & $2-3[2]$ & $0.0277^{*}$ \\
\hline Total FIM & $23-124[98]$ & 24-95 [60] & 0.3782 \\
\hline FIM: motor function & $16-89[63]$ & $14-70[35]$ & 0.3782 \\
\hline FIM: cognitive function & $7-35[35]$ & $10-28[25]$ & $0.036^{*}$ \\
\hline
\end{tabular}

(): mean, []: median, *: Chi-squared test, P < 0.05, Brs: Brunnstrom stage, F: female, FIM: functional independence measure, FMA: Fugl-Meyer assessment, HAL: hybrid assistive limb, M: male, SIAS: stroke impairment assessment set.

effects of visual feedback from the position marker of center of gravity displayed on the monitor screen and the posture monitor are considered to contribute to the excitability of the intracortical network. Such mechanistic actions of HAL enhance the excitability of the corticospinal tract and promote the structure of the intracortical network, and are consistent with the mechanisms underlying acute-phase recovery from motor paralysis proposed by Swayne et al. ${ }^{13}$

To the best of our knowledge, this is the first study to investigate the suitability of HAL. Our results demonstrated that the effects of physical therapy using HAL were greater in patients with high visual-cognitive function and communication ability. This was presumably due to the patient being able to understand the therapist's explanations, consequently leading to efficient extraction of interactive feedback through HAL, and due to the patient being able to receive visual feedback from the monitor.

Physical therapy that uses HAL requires greater understanding and acceptance of unusual machinery than typical treatments. Effective training cannot be performed when there is refusal towards such unusual therapy or reduced willingness due to a lack of understanding. When performing interventions using HAL, screening tests such as FIM and SIAS that can assess various aspects including peripheral function may be warranted.

\section{Limitations}

The study population included only patients who underwent HAL intervention in the acute phase, and not those in the recovery or chronic phases. In addition, this study did not compare patients who performed training using HAL with patients who only performed normal training without the HAL, and whether training with HAL indeed has benefits over training without HAL thus remains unclear. This study included cases that had undergone only two or three sessions. The reasons were patient demands or early functional recovery. While these cases might not represent the effects of HAL, we judged the results as suggesting the suitability of training programs using HAL. Tests that assess cognitive function and attention or motivation function such as the mini-mental state examination (MMSE), revised Hasegawa's dementia scale (HDS-R), clinical assessment for attention (CAT), clinical assessment for spontaneity (CAS), and profile of mood states (POMS) were not conducted, and detailed evaluations of various functions have yet to be performed. In addition, our study population was small and motor function during intervention did not differ 
significantly between the two HAL suitability groups, but a significant difference may be extracted by increasing the study population.

\section{Conclusion}

Training using HAL in patients with a central nervous system injury during the acute phase does not entail pain and exhibits positive effects in those with high visuospatial perception and communication ability. To predict the suitability of HAL for usage in intervention, functional assessment scores (FIM and SIAS) including peripheral items appear useful.

\section{Conflicts of Interest Disclosure}

None declared. All authors who are members of the Japan Neurosurgical Society have registered online self-reported COI disclosure statement forms.

\section{References}

1) Hsu WY, Cheng CH, Liao KK, Lee IH, Lin YY: Effects of repetitive transcranial magnetic stimulation on motor functions in patients with stroke: a metaanalysis. Stroke 43: 1849-1857, 2012

2) Hsu SS, Hu MH, Wang YH, Yip PK, Chiu JW, Hsieh CL: Dose-response relation between neuromuscular electrical stimulation and upper-extremity function in patients with stroke. Stroke 41: 821-824, 2010

3) Thieme H, Mehrholz J, Pohl M, Behrens J, Dohle C: Mirror therapy for improving motor function after stroke. Stroke 44: e1-e2, 2013

4) Veerbeek JM, van Wegen E, van Peppen R, van der Wees PJ, Hendriks E, Rietberg M, Kwakkel G: What is the evidence for physical therapy poststroke? A systematic review and meta-analysis. PLoS One 9: e87987, 2014

5) Aach M, Cruciger O, Sczesny-Kaiser M, Höffken O, Meindl RCh, Tegenthoff M, Schwenkreis P, Sankai Y, Schildhauer TA: Voluntary driven exoskeleton as a new tool for rehabilitation in chronic spinal cord injury: a pilot study. Spine J 14: 2847-2853, 2014

6) Cruciger O, Schildhauer TA, Meindl RC, Tegenthoff M, Schwenkreis P, Citak M, Aach M: Impact of locomotion training with a neurologic controlled hybrid assistive limb (HAL) exoskeleton on neuropathic pain and health related quality of life (HRQoL) in chronic SCI: a case study. Disabil Rehabil Assist Technol 10: 1-6, 2014 [Epub ahead of print]

7) Tsukahara A, Hasegawa Y, Eguchi K, Sankai Y: Restoration of gait for spinal cord injury patients using HAL with intention estimator for preferable swing speed. IEEE Trans Neural Syst Rehabil Eng 23: 308-318, 2015

8) Sakakima H, Ijiri K, Matsuda F, Tominaga H, Biwa T, Yone K, Sankai Y: A newly developed robot suit hybrid assistive limb facilitated walking rehabilitation after spinal surgery for thoracic ossification of the posterior longitudinal ligament: a case report. Case Rep Orthop 2013: 621405, 2013

9) Watanabe H, Tanaka N, Inuta T, Saitou H, Yanagi H: Locomotion improvement using a hybrid assistive limb in recovery phase stroke patients: a randomized controlled pilot study. Arch Phys Med Rehabil 95: 2006-2012, 2014

10) Kawamoto H, Kamibayashi K, Nakata Y, Yamawaki K, Ariyasu R, Sankai Y, Sakane M, Eguchi K, Ochiai N: Pilot study of locomotion improvement using hybrid assistive limb in chronic stroke patients. BMC Neurol 13: 141, 2013

11) Nilsson A, Vreede KS, Häglund V, Kawamoto $H$, Sankai Y, Borg J: Gait training early after stroke with a new exoskeleton-the hybrid assistive limb: a study of safety and feasibility. J Neuroeng Rehabil 11: 92,2014

12) Ueba $T$, Hamada $O$, Ogata $T$, Inoue $T$, Shiota $E$, Sankai Y: Feasibility and safety of acute phase rehabilitation after stroke using the hybrid assistive limb robot suit. Neurol Med Chir (Tokyo) 53: 287-290, 2013

13) Swayne OB1, Rothwell JC, Ward NS, Greenwood RJ: Stages of motor output reorganization after hemispheric stroke suggested by longitudinal studies of cortical physiology. Cereb Cortex 18: 1909-1922, 2008

Address reprint requests to: Yasushi Takagi, Department of Neurosurgery, Kyoto University, 54 Shougoinkawaramachi, Sakyo-ku, Kyoto 604-0901, Japan. e-mail: ytakagi@kuhp.kyoto-u.ac.jp 